\title{
Assessing the Montevideo Interpretation of Quantum Mechanics
}

\author{
Jeremy Butterfield \\ Trinity College, Cambridge CB2 1TQ: jb56@cam.ac.uk \\ Published in Studies in the History and Philosophy of Modern Physics, May 2014 \\ (online)
}

Sunday 15 June 2014

\begin{abstract}
This paper gives a philosophical assessment of the Montevideo interpretation of quantum theory, advocated by Gambini, Pullin and co-authors. This interpretation has the merit of linking its proposal about how to solve the measurement problem to the search for quantum gravity: namely by suggesting that quantum gravity makes for fundamental limitations on the accuracy of clocks, which imply a type of decoherence that 'collapses the wave-packet'.

I begin (Section 2) by sketching the topics of decoherence, and quantum clocks, on which the interpretation depends. Then I expound the interpretation, from a philosopher's perspective (Sections 3, 4 and 5). Finally, in Section 6, I argue that the interpretation, at least as developed so far, is best seen as a form of the Everett interpretation: namely with an effective or approximate branching, that is induced by environmental decoherence of the familiar kind, and by the Montevideans' 'temporal decoherence'.
\end{abstract}

Keywords: Montevideo interpretation; quantum clocks; decoherence; Everett interpretation 


\section{Contents}

1 Introduction $\quad 3$

2 The landscape $\quad 3$

2.1 Collapse, Everett, decoherence - and gravity . . . . . . . . . . . . 3

2.1.1 The collapse of the wave-packet . . . . . . . . . . . . 4

2.1.2 Everett, decoherence and patterns . . . . . . . . . . . 4

2.1.3 Gravity?. . . . . . . . . . . . . . . . 8

2.2 Quantum clocks ....................... . . 9

2.3 The road ahead . . . . . . . . . . . . . . . . . . 11

3 Evolution with respect to a real clock 12

4 The meanings and roles of a mixture $\quad 15$

4.1 Two roles, two approaches and two debates . . . . . . . . . . 15

4.1.1 Can we ignore small amplitudes? . . . . . . . . . . . . . . . . 17

4.1 .2 Is vagueness a virtue or a vice? . . . . . . . . . . . . . . 17

4.2 Montevideo's mixtures . . . . . . . . . . . . . . . . . . 19

5 Throwing away the ladder $\quad 20$

5.1 Four proposals . . . . . . . . . . . . . . . . 20

5.1.1 Heuristic arguments . . . . . . . . . . . . . . 21

5.1 .2 Fundamental decoherence . . . . . . . . . . . . . 21

5.1.3 Fundamental decoherence allied with environmental decoherence 22

5.1.4 Robustness . . . . . . . . . . . . . . . . . 22

5.2 The fifth proposal: about exactly when is there an outcome . . . . . . 23

6 Everett en fin 24

7 References $\quad 25$ 


\section{Introduction}

This paper gives a philosophical assessment of a recent proposed interpretation of quantum theory, advocated by Gambini, Pullin and co-authors, and called by them 'the Montevideo interpretation'. (So I will dub these authors 'the Montevideans'.) Although the interpretation is bound to be controversial, it has the merit of linking its proposed solution to the measurement problem to the search for quantum gravity: in short, by suggesting that quantum gravity makes for fundamental limitations on the accuracy of clocks, which imply a specific temporal type of decoherence that 'collapses the wave-packet'. For it is surely a merit to link debate about quantum foundations to the search for new physics, even speculative new physics.

I therefore begin by sketching the standard topics on which the interpretation depends (Section 2). Then I expound the interpretation, from a philosopher's perspective (Sections 3, 4 and 5). ${ }^{1}$ Finally, in Section 6, I argue that the interpretation, at least as developed so far, is best seen as a form of the Everett interpretation: namely with an effective or approximate branching, that is induced by environmental decoherence of the familiar kind, and by the Montevideans' 'temporal decoherence'.

\section{The landscape}

I introduce the measurement problem and quantum clocks, in Sections 2.1 and 2.2 respectively. Then we will be ready for a prospectus about the Montevideo interpretation (Section 2.3).

\subsection{Collapse, Everett, decoherence - and gravity}

I will recall the relevant aspects of the measurement problem, by briefly sketching: the collapse of the wave-packet (Section 2.1.1), the Everett approach and decoherence (Section 2.1.2), and how the problem may be altered by considering gravity (Section 2.1.3). Later, I will return to these aspects in more detail: the first three in Sections 4.1 and 5.2, and the last in Section 5.1.

\footnotetext{
${ }^{1}$ This interpretation has been developed in about a dozen papers over the last ten years. As I see matters, the main ones are: Gambini, Garcia Pintos and Pullin (2010, 2011, 2011a), Gambini, Porto and Pullin (2006, 2007, 2008), Gambini and Pullin (2007, 2009, 2009a). For the way in which considerations of quantum gravity, especially the 'problem of time' in quantized general relativity, have motivated the interpretation, cf. Gambini, Porto, Pullin and Torterolo (2009) and Gambini and Pullin (2009a). But my summary will draw on just a few aspects of Gambini, Porto and Pullin (2007), and Gambini and Pullin (2009, 2009a); where I would recommend a philosopher to begin their reading. The papers are also available at a website: http://www.montevideointerpretation.org/.
} 


\subsubsection{The collapse of the wave-packet}

Let us begin with the orthodox - or at least, traditional and minimalist - approach to securing that measurements have definite outcomes. 'The collapse of the wavepacket' refers to an irreducibly indeterministic change in the state of a quantum system, contravening the deterministic and continuous evolution prescribed by the Schroedinger equation. Anyone who advocates such a collapse, as a bona fide physical process that occurs to an isolated system, faces several questions. Three of the most pressing are as follows. Under exactly what conditions does the collapse occur? What determines the physical quantity (the basis) with respect to which it occurs? How does it mesh with relativity? Good questions, which went largely un-addressed by quantum theory's founding fathers: but which in recent decades have been addressed by a great deal of good work. Section 4.1 will mention one main line, the dynamical reduction programme of Ghirardi and others.

\subsubsection{Everett, decoherence and patterns}

The Everett or many-worlds interpretation proposes to reconcile quantum theory's deterministic evolution of the quantum state with the apparent collapse of the wave packet, i.e. with measurements having definite outcomes with various frequencies, by saying that measurement processes involve a splitting of the universe into branches. Obviously, this proposal has to face its own versions of the murky questions just mentioned: about the conditions under which a branching occurs, how we should understand branching, and how branching can mesh with relativity. Murky indeed. So it is hardly surprising that this interpretation has traditionally been regarded as vaguer and more controversial than others. Thus Bell, in his masterly (1986) introduction to interpreting quantum theory, wrote that it 'is surely the most bizarre of all [quantum theory's possible interpretations]' and seems 'an extravagant, and above all extravagantly vague, hypothesis. I could almost dismiss it as silly' (pp. 192, 194).

But I submit since Bell wrote, Everettians have made major improvements to their interpretation. In my opinion, there have been two main improvements relevant to our purposes: which I will label "Decoherence' and 'Patterns'.

\subsubsection{Decoherence :}

Although the fundamental ideas of decoherence were established in the early years (and were clear to maestros such as Heisenberg, Mott and Bohm), detailed models were only developed from about 1980; (Schlosshauer (2008) is an excellent recent survey).

\footnotetext{
${ }^{2}$ I set aside a third improvement, viz. various arguments justifying, from an Everettian perspective, the orthodox (Born-rule) form of quantum probabilities. All three improvements have been developed in many papers over the last twenty years. Some of the latest work is in Saunders et al. (2010), and by Wallace $(2012,2012 \mathrm{a})$. The first of these also contains penetrating critical assessments of all three improvements by non-Everettians.
} 
Recall that 'decoherence' means, broadly speaking, the diffusion of quantum coherence from the system to its environment. This is the fast and ubiquitous process whereby, for appropriate physical quantities, the interference terms in probability distributions, that are characteristic of the difference between a superposition and a mixture, diffuse from the system to its environment. In a bit more detail: at the end of the decoherence process, the quantum probabilities for any quantity on the system are as if the system is in one or other of a definite set of states. In many models of how a system (such as a dust-particle) interacts with its environment (such as air molecules), this set consists of coherent states: states which are sharply peaked for both position and momentum, so that a system in any such state is presumably nearly definite in both position and momentum. (But the distributions have enough spread so as to obey the Uncertainty Principle's veto on simultaneous precise values for position and momentum.)

For our purposes, decoherence has two important features. The first is a kind of imprecision. That sounds like a defect; but I shall maintain - especially later, in Sections 4.1 and 6-that this imprecision is, for the Everettian, a merit. (Here I follow in the footsteps of some avowed Everettians, such as Saunders and Wallace.) So I will call this feature 'flexibility'.

Thus we expect the classical physical description of the world to be vindicated by quantum theory - but only approximately. Only some subset of quantities should have definite values. And maybe that subset should only be specified contextually, even vaguely. And maybe the values should only be definite within some margins of error, even vague ones. Decoherence secures this sort of flexibility. For the selection of the quantity that is preferred in the sense of having definite values (relative to a branch) is made by a dynamical process - whose definition can be legitimately varied in several ways. Three examples: the definitions of the system-environment boundary, and of the time at which the interaction ends, and of what counts as a state being 'sharply peaked' for a quantity, can all be varied.

The second feature is that decoherence does not just by itself solve the measurement problem. More precisely: it does not imply that the system is in one of the set of states (typically coherent states). It implies only that the quantum probabilities are as if the system were in one. Furthermore, the theory implies that the system is in fact not in one of those states (on pain of contradicting the original hypothesis that the total system-plus-environment is in a superposition, not a mixture). This feature is wellknown, and has been given various names, especially 'the problem of outcomes', 'the problem of improper mixtures' (following a jargon of D'Espagnat) and the 'the problem of replacing 'and' by 'or' (following a jargon of Bell); (cf. my discussion of (Outcome) in Section 4.1).

To put this feature vividly, in terms of Schroedinger's cat: at the end of the decoherence process, the quantum state still describes two cats, one alive and one dead. It is just that the two cats are correlated with very different microscopic states of the surrounding air molecules. For example: an air molecule will bounce off a wagging 
upright tail, and a stationary downward one, in different directions! Since one's overall aim is to solve the measurement problem, this feature is usually considered a defect, not a merit, of decoherence. But we will now see how the Everettians' second main development may turn it into a merit ...

\subsubsection{Patterns :}

The second development is the application to the problems of quantum ontology, especially Schroedinger's cat, of the philosophical idea that the objects in 'higher-level' ontology, e.g. a cat, are not some kind of aggregate (e.g. a mereological fusion) of lower-level objects, but are dynamically stable patterns of them, of a special typewhich type being spelt out by what we believe about objects of that kind. This idea is often associated with "functionalism" in the philosophy of mind (e.g. Dennett 1991).

Some prominent Everettians, such as Saunders and Wallace, maintain that it snatches victory from the jaws of defeat: the defeat, just mentioned, that decoherence apparently does not by itself solve the measurement problem. The idea is that the final quantum state's describing two cats, one alive and one dead, is a matter of the state encoding two patterns - and the description is entirely right.

This becomes a bit clearer if we adopt a specific representation of the quantum state, for example position. Then, roughly speaking: the final state is a wave-function on the cat's classical configuration space, with two peaks: one peak over some classical configurations each corresponding to a live cat, e.g. with a wagging upright tail, the other peak over some classical configurations corresponding to a dead cat, e.g. with a stationary downward tail. But in that case: according to the idea of cats as patterns, the quantum state does indeed represent two cats.

In other words: we see that we should take the measurement problem to be solved by exactly what decoherence secures: a final state describing a living cat and a dead one. In brief: the philosophical idea of higher-level objects as patterns vindicates the Everettian vision of a multiplicity of objects.

It is worth stressing (as Wallace, for one, does) that this line of thought is independent of quantum theory's details; and so it is also independent of its various weird features (e.g. non-locality). The point is closely analogous to one which we all unhesitatingly endorse for several other physical theories. Namely, theories in which states can be added together to give a sum-state, while the component states are dynamically isolated, or nearly so (i.e. do not influence each other). Examples include the theory of water-waves, or electromagnetism. So, says Wallace, we should also endorse it in quantum theory, and accept that there are two cats.

For example: the water in Montevideo harbour can get into a state which we describe as, e.g. a wave passing through the harbour's centre heading due West; or into a state which we describe as a wave passing through the centre heading due North; or into a state which is the sum of these. But do we face a 'Montevideo water paradox'? Do we agonize about how the Montevideo harbour water-system can in one place (viz. 
the harbour's centre) be simultaneously both Westward and Northward? Of course not. Rather, we say that waves are higher-level objects, patterns in the water-system; and that there are two waves, with the contrary properties, one Northward and one Westward. Similarly for the electromagnetic field in a certain region, and e.g. pulses of laser light travelling in different directions across it.

And similarly, says Wallace, about the quantum wave-functions defined on the classical configuration space. There is a state with two cats, one alive and one dead. And of course, there are also myriad other states, the vast majority of which do not represent macroscopic objects (patterns!) which we might recognize (as cats or as dogs or as puddles or as mud or ...) — or even sums of these.

\subsubsection{Summing up :}

Let me sum up these two developments, Decoherence and Patterns. Nowadays, some Everettians propose to combine the physics of decoherence with the philosophical ("functionalist") idea that objects in 'higher-level' ontology are dynamically stable patterns of lower-level objects. This suggests that the proverbial Schroedinger's cat measurement involves an approximate and emergent splitting, after which there really are two cats (or two broad kinds of cat), since the total wave-function is peaked over two distinctive kinds of pattern in the classical configuration space: the legs, tail and body all horizontal, still and cool ('dead'); and the legs and tail vertical, moving and warm ('alive').

I think these Everettians would admit how hard it is to get one's mind around the central idea: the dizzying vision of decoherence processes yielding a continual, but approximate and emergent, branching of the universe that:

(a): meshes fundamentally with relativity, so that there is no absolute time ordering to the splitting; and

(b): is to be combined with almost all objects - not just macroscopic objects like cats, tables and stars, but anything that classical physics successfully describes as having a spatial trajectory etc.: for example, large molecules-being treated as patterns; or better: being treated as the quantum state being peaked above such patterns in a classical configuration space.

I said 'some Everettians', since I do not think there is unanimity among them. One main question is to what extent they should try to make this dizzying vision more precise in general terms, perhaps by finding a new mathematical language to describing the emergent branching. Thus Deutsch, in the course of his striking appeal (2010) to fellow-Everettians to stop defending the interpretation against accusations and rivals, and instead explore the new physics that it promises to contain, admits that the exploration will be very challenging, since no one has yet given a precise mathematical descriptions of (even toy models of) this branching structure (2010, p. $546)$.

On the other hand, Saunders and Wallace (at least as I read them) do not think any such precise description could, or should, be possible. Nor is it desirable: vagueness 
about when branching occurs is a merit - recall the label 'flexibility'. They draw an analogy which may be helpful: between (a) times as understood in the 'block-universe' view of time suggested by relativity theory (also known in philosophy as: the 'B-theory' of time), and (b) worlds or branches as understood by the Everettian. Recall that the advocate of the block-universe says:

(i): reality is four-dimensional, and slices across it are in principle arbitrary and artefactual (especially in relativity theory, with no absolute simultaneity); but also

(ii): for describing the history of the universe - and in particular, in physics, for doing dynamics - only a small subset of slices will be useful; though the criteria to select that subset will be a bit approximate.

Then the proposed analogy is as follows. Similarly, the Everettian says:

(i'): the 'slicing' of reality by choosing a basis in Hilbert space is in principle arbitrary and artefactual; but also

(ii'): for describing the history of the universe - and in physics, for doing the approximate and emergent dynamics of a world - only a small subset of bases will be useful; though the criteria to select that subset will be a bit approximate (since decoherence gives no absolute criterion for a system-environment split, or for when interference terms are small enough).

In short: the analogy is between arbitary slicings of spacetime, and arbitrary choices of basis in Hilbert space. Both are precise; in both cases, for doing dynamics, only a small subset will be useful; in both cases, the criteria to select that subset will be a bit approximate. ${ }^{3}$

So this is very much an open debate among the Everettians. ${ }^{4}$ But I can duck out of pursuing it: for my purposes in assessing the Montevideans, it will be enough that some Everettians maintain that the vagueness of the branching is a merit.

\subsubsection{Gravity?}

I will make two remarks about how considerations about gravity might contribute to solving the measurement problem. Both are very programmatic and brief, reflecting the fact that we do not have a satisfactory unified treatment of quantum theory and gravity; nor even a consensus about what it would look like. ${ }^{5}$ But they are worth making, to set the stage for the Montevideo interpretation.

First: in the controversies about what a quantum theory of gravity should look like, one central and long-established theme has been the conflict between the way general relativity treats space and time, namely as dynamical (variable, and interacting with matter and radiation), and the way other theories, both classical and quantum, treat

\footnotetext{
${ }^{3}$ For more details, cf. Wallace (2001); or more briefly, Butterfield (2002, Sections 7 and 8).

${ }^{4}$ And of course, it echoes a wider debate about whether fundamental physical theories must be precise. In this context, I recommend Kent's (2010) critique of the Everett interpretation.

${ }^{5}$ For philosophical introductions of the issues involved, cf. e.g. Butterfield and Isham (2001), and Rovelli (2006).
} 
them, namely as non-dynamical (also called: 'fixed' or 'background'). Besides, in one main approach to quantum gravity, viz. the quantization of canonical (i.e. Hamiltonian) formulations of general relativity, this conflict is especially striking, indeed severe, as regards the treatment of time rather than space: and accordingly, it is called 'the problem of time'. Though I shall not pursue this problem, I should emphasize that the Montevideo interpretation grew out of the Montevideans' engagement with it. For details, cf. e.g. Gambini, Porto, Pullin and Torterolo (2009) and Gambini and Pullin (2009a).

Second: there are various proposals that gravity might help solve the measurement problem. One example is Penrose's proposal that gravity drives the collapse of the wave-packet; or a little more precisely: that a sufficiently large difference in the gravitational self-energy of two configurations of masses renders superpositions of such configurations unstable. The Montevideans' proposal will be different: the idea will be that quantum gravity implies fundamental limitations on clocks. To set the stage, the next Section briefly discusses quantum clocks.

\subsection{Quantum clocks}

The treatment of time in quantum theory is a large and controversial subject - though fortunately, not as large and controversial as the measurement problem as a whole! But in the last few decades, the situation has become clearer through the work of many people on such topics as the time-energy uncertainty principle, and the conditions for the existence of a time operator. ${ }^{6}$

To prepare for the later discussion, I need here only to sketch two themes about quantum clocks. Both concern limits to their resolution, arising from uncertainty principles. The first theme is elementary and general, and so I can give some details; the second involves detailed arguments about how certain ideal clocks might be constructed, and I will just cite the results the Montevideans will appeal to. These themes are:

(i): the time-energy uncertainty principle constrains the resolution of clocks, however they might be constructed: roughly, narrowness in the energy distribution worsens the resolution of a clock;

(ii): applying uncertainty principles to certain ideal clocks implies that mass constrains the resolution: roughly, a lower mass worsens the resolution.

(i): The time-energy uncertainty principle:

I shall assume that here, 'time' refers to a dynamical variable of the quantum system, whose value, especially expectation value, serves as a clock-pointer, i.e. to measure time. Thus in principle, every non-stationary quantity $A$ defines for any state $\rho$ a characteristic time $\tau_{\rho}(A)$ in which $\langle A\rangle$ changes 'significantly'. For example: if $A=Q$,

\footnotetext{
${ }^{6}$ Butterfield (2012) is a philosophical introduction, emphasizing the first topic, especially the work of Busch, Hilgevoord and Uffink. For the whole subject of time in quantum theory, Muga et al. (2008) is an excellent collection; for a glimpse of work since then, I recommend Yearsley (2011).
} 
and $\rho$ is a wave packet, say $\rho=|\psi\rangle\langle\psi|$, then $\tau_{\rho}(A)$ could be defined along the lines: the time for the bulk of the wave packet to shift by its width. This assumption is not controversial. It corresponds to what Busch in his excellent survey (2008) calls 'intrinsic time' (and in his earlier (1990, 1990a), 'dynamical time').

With this notion of time, we can easily derive what is perhaps the best-known timeenergy uncertainty principle, the Mandelstam-Tamm principle. Namely, we define the characteristic time for any quantity $A$ by

$$
\tau_{\rho}(A):=\Delta_{\rho} A /\left|d\langle A\rangle_{\rho} / d t\right|
$$

and readily deduce (e.g. Messiah 1965, p. 320)

$$
\tau_{\rho}(A) \Delta_{\rho}(H) \geq \frac{1}{2} \hbar
$$

An easy example is given by a free particle in a pure state $\psi$ with a sharp momentum i.e. $\Delta_{\psi} P<<\left|\langle P\rangle_{\psi}\right|$. We can deduce that $\Delta_{\psi} Q(t) \approx \Delta_{\psi} Q\left(t_{0}\right)$, i.e. the wave-packet spreads slowly; so that the Mandelstam-Tamm time $\tau_{\rho}(Q)$, as defined by eq 2.1 , is indeed the time it takes for the packet to propagate a distance equal to its standard deviation.

But to discuss the resolution of a quantum clock, we need instead the example of the lifetime of a property represented by a projector $\hat{P}$. Let us define $p(t):=$ $\left\langle\psi_{0}\left|U_{t}^{-1} \hat{P} U_{t}\right| \psi_{0}\right\rangle$; where of course $U_{t}:=\exp (-i t H / \hbar)$, so that $p(t)$ is the probability of "having" $\hat{P}$ at $t$. Then the Mandelstam-Tamm uncertainty relation gives

$$
\left|\frac{d p}{d t}\right| \leq \frac{2}{\hbar}\left(\Delta_{\psi_{0}} H\right)[p(1-p)]^{\frac{1}{2}}
$$

Integration with initial condition $p(0) \equiv 1$ (i.e. $\hat{P}$ actual at $t=0$ ) yields

$$
p(t) \geq \cos ^{2}\left(t\left(\Delta_{\psi_{0}} H\right) / \hbar\right) \text { for } 0<t<\frac{\pi}{2} \frac{\hbar}{\left(\Delta_{\psi_{0}} H\right)}
$$

so that if we define the lifetime $\tau_{\hat{P}}$ of the property $\hat{P}$ as a "half-life", i.e. by $p\left(\tau_{\hat{P}}\right):=\frac{1}{2}$, we deduce

$$
\tau_{\hat{P}} \cdot \Delta_{\psi_{0}} H \geq \frac{\pi \hbar}{4}
$$

The general point is that the rate of change of a property of the system decreases with increasing sharpness of the energy: and in the limit of an energy eigenstate, all quantities have, of course, stationary distributions.

It is straightforward to apply the Mandelstam-Tamm inequality eq. 2.5 to get a lower bound on the resolution of a quantum clock. The general idea of such a clock is that it is a sequence $\psi_{1}, \psi_{2}, \ldots$ of orthogonal clock-pointer states that are occupied at equi-spaced times $t_{1}, t_{2}, \ldots$; so the clock resolution is $\delta t:=t_{k+1}-t_{k}$. Thus suppose the clock-pointer is the mean position of a wave packet. Then eq. 2.5, together with 
the constraint that the resolution be greater than the Mandelstam-Tamm time, i.e. $\delta t \geq \tau_{\psi}(Q)$, implies

$$
\delta t \geq \tau_{\psi}(Q) \geq \frac{\pi \hbar}{4} \frac{1}{\Delta_{\psi} H} .
$$

So the sharper the energy, the worse the resolution: more exactly, the higher is the resolution's lower bound. ${ }^{7}$

(ii): A constraint from mass:

There are some heuristic arguments that apply uncertainty principles (of positionmomentum, as well as time-energy) to certain ideal clocks (e.g. bouncing light between two mirrors), and conclude that there are fundamental limits on the clocks' resolution: limits additional to those sketched in (i) above.

Thus in a famous analysis, Salecker and Wigner (1957) argued that the mass of a clock limited its accuracy: specifically, if $T$ is the time-interval to be measured by a clock of mass $M$, then there must be an uncertainty $\delta T$ proportional to $\sqrt{ } T / M$. This analysis suggests that one can attain arbitrary accuracy by a sufficiently massive clock. But Ng and van Dam (1994) argued that in general the mass needs to be confined, and that therefore the attainable mass, and so accuracy, is limited by the onset of the formation of a black hole (!). I need not go into the details. Here it will suffice to say that the Montevideans will endorse this line of argument (Section 5.1.1).

\subsection{The road ahead}

So much by way of sketching the landscape. From now on, I turn to assess the Montevideo interpretation of quantum theory. As I said in Section 1, the proposal is to solve the measurement problem by combining decoherence (as in Section 2.1) with a fundamental limitation on the accuracy of quantum clocks (as in Section 2.2) which is suggested by some heuristic considerations about quantum gravity.

The plan is as follows. I will first summarize the Montevideans' analysis of how, in orthodox quantum theory, inaccurate clocks make for mixed quantum states (Section 3). Then I discuss these states' interpretation, by comparison with the mixed states obtained in two other approaches, viz. decoherence by the environment and dynamical reduction (Section 4). In Section 5, I report the Montevideans' main proposals: that considerations about quantum gravity make for a fundamental limitation on the accuracy of quantum clocks, which solves the measurement problem - in effect, by answering objections that are made to the mixed states obtained by environment-induced decoherence. Finally, in Section 6, I will urge that the Montevideo interpretation is best seen as a version of the Everett interpretation, with an effective or approximate branching. It will be clear that the discussion becomes more controversial, as we proceed:

\footnotetext{
${ }^{7}$ There is a great deal more to say here, even without going into details about clocks' construction as the arguments cited in (ii) will do. For there is a large literature on various versions of the time-energy uncertainty principle (Busch 2008): for example, Butterfield (2012) advertises how the Hilgevoord-Uffink uncertainty principles have advantages over the usual ones.
} 
one can 'get off the bus' at various stops. That is: in the present state of theoretical and experimental knowledge, the later proposals remain unproven-but nonetheless interesting!

\section{Evolution with respect to a real clock}

The Montevideans' first contribution is a natural sequel to Section 2.2. For it is an analysis within orthodox quantum theory of what the phrase, "the probability of a given outcome, for a measurement on a given state at a given time', really means, when one takes seriously that the 'given time' is as read by a quantum quantity. (This Section summarizes part of Section II of Gambini, Porto and Pullin (2007), and follows their notation.)

We envisage measuring a quantity $\hat{O}$ on a quantum system, when a quantity, $\hat{T}$ say, on another quantum system ('the clock') has value ' $T$ '. Both systems evolve in the orthodox unitary (and non-interacting) manner with respect to the background time $t$. We work in the Heisenberg picture (with respect to $t$ ), and allow for continuous spectra. So the projector for the clock system for the value $T$ lying in the interval $\left[T_{0}-\Delta T, T_{0}+\Delta T\right]$ is

$$
P_{T_{0}}(t):=\int_{T_{0}-\Delta T}^{T_{0}+\Delta T} d T \Sigma_{k}|T, k, t\rangle\langle T, k, t|
$$

where: (i) the $t$ in the bra and ket signal the use of the Heisenberg picture; (ii) $k$ represents the eigenvalues of some operators that together with $\hat{T}$ form a complete set; and (iii) if the $k$ have a partly continuous spectrum the sum over $k$ should be appropriately replaced by an integral.

Similarly the projector for the measured system for the value $O$ lying in the interval $\left[O_{0}-\Delta O, O_{0}+\Delta O\right]$ is

$$
P_{O_{0}}(t):=\int_{O_{0}-\Delta O}^{O_{0}+\Delta O} d O \Sigma_{j}|O, j, t\rangle\langle O, j, t|
$$

where $j$ represents eigenvalues of operators that together with $\hat{O}$ form a complete set.

Then the (orthodox Born-rule) conditional probability that $\hat{O}$ takes value $O_{0}$ given that the clock indicates time $T_{0}$ is

$\mathcal{P}\left(O \in\left[O_{0}-\Delta O, O_{0}+\Delta O\right] \mid T \in\left[T_{0}-\Delta T, T_{0}+\Delta T\right]\right)=\frac{\lim _{\tau \rightarrow \infty} \int_{-\tau}^{\tau} d t \operatorname{Tr}\left(P_{O_{0}}(t) P_{T_{0}}(t) \rho P_{T_{0}}(t)\right)}{\lim _{\tau \rightarrow \infty} \int_{-\tau}^{\tau} d t \operatorname{Tr}\left(P_{T_{0}}(t) \rho\right)}$

where: (i) $\rho$ is the (Heisenberg-picture, so $t$-constant) state of the combined systemplus-clock; (ii) the integrals over all $t$ on the right hand side reflect our ignorance about when in the background time $t$ the clock takes the value $T_{0}$; (iii) we envisage 
that $\Delta T$ is chosen much smaller than any characteristic time scales of the problem (in particular, the time-interval between successive measurements, if we were to consider multiple measurements); and (iv) we envisage that $\hat{T}$ is so chosen that it does not take the same value twice (at least in the lifetime of the experiment).

Our aim now is to relate this in-principle expression to the usual expression for the Born-rule probability that $\hat{O}$ takes value $O_{0}$ at time $t$ : which is, in the more familiar Schroedinger picture and an obvious notation:

$$
\mathcal{P}\left(O_{0} \mid t\right)=\frac{\operatorname{Tr}\left(P_{O_{0}}(0) \rho(t)\right)}{\operatorname{Tr}(\rho(t))} .
$$

To this end, we now assume that the state is a product of the observed system and the clock, and that they never interact. So $\rho=\rho_{\mathrm{sys}} \otimes \rho_{\mathrm{cl}}$ and $U=U_{\mathrm{sys}} \otimes U_{\mathrm{cl}}$. Then the conditional probability eq. 3.3 is equal to:

$$
\frac{\lim _{\tau \rightarrow \infty} \int_{-\tau}^{\tau} d t \operatorname{Tr}\left(U_{\mathrm{sys}}(t)^{\dagger} P_{O_{0}}(0) U_{\mathrm{sys}}(t) U_{\mathrm{cl}}(t)^{\dagger} P_{T_{0}}(0) U_{\mathrm{cl}}(t) \rho_{\mathrm{sys}} \otimes \rho_{\mathrm{cl}}\right)}{\lim _{\tau \rightarrow \infty} \int_{-\tau}^{\tau} d t \operatorname{Tr}\left(P_{T_{0}}(t) \rho_{\mathrm{cl}}\right) \operatorname{Tr}\left(\rho_{\mathrm{sys}}\right)}
$$

which is equal to

$$
\frac{\lim _{\tau \rightarrow \infty} \int_{-\tau}^{\tau} d t \operatorname{Tr}\left(U_{\text {sys }}(t)^{\dagger} P_{O_{0}}(0) U_{\text {sys }}(t) \rho_{\text {sys }}\right) \operatorname{Tr}\left(U_{\mathrm{cl}}(t)^{\dagger} P_{T_{0}}(0) U_{\mathrm{cl}}(t) \rho_{\mathrm{cl}}\right)}{\lim _{\tau \rightarrow \infty} \int_{-\tau}^{\tau} d t \operatorname{Tr}\left(P_{T_{0}}(t) \rho_{\mathrm{cl}}\right) \operatorname{Tr}\left(\rho_{\mathrm{sys}}\right)} .
$$

We now define

$$
\mathcal{P}_{t}(T):=\frac{\operatorname{Tr}\left(P_{T_{0}}(0) U_{\mathrm{cl}}(t) \rho_{\mathrm{cl}} U_{\mathrm{cl}}(t)^{\dagger}\right)}{\int_{-\infty}^{\infty} d t \operatorname{Tr}\left(P_{T_{0}}(t) \rho_{\mathrm{cl}}\right)}
$$

which obeys $\int_{-\infty}^{\infty} d t \mathcal{P}_{t}(T)=1$, and represents a probability density of $t$ at given $T$; (not 'of $T$ at given $t$ ', despite the notation).

We also define an "effective" density matrix (of the observed system) as a function of $T$, by

$$
\rho_{\text {eff }}(T):=\int_{-\infty}^{\infty} d t U_{\text {sys }}(t) \rho_{\text {sys }} U_{\text {sys }}(t)^{\dagger} \mathcal{P}_{t}(T)
$$

With these definitions, it is easy to verify, by noting that

$$
\operatorname{Tr}(\rho(T))=\int_{-\infty}^{\infty} d t \mathcal{P}_{t}(T) \operatorname{Tr}\left(\rho_{\mathrm{sys}}\right)=\operatorname{Tr}\left(\rho_{\mathrm{sys}}\right)
$$

\footnotetext{
${ }^{8}$ I thank Gordon Fleming for pointing out that in eq.s 3.3 to 3.6, one needs in general a ratio of limits, so as to secure equality with the conditional probability on the left hand side of eq. 3.3. In fact, Gambini et al (ibid.) write a limit of a ratio, i.e. one $\tau$ limit governing the ratio of two integrals: which is of course equal to the ratio of limits, provided both numerator and denominator have a limit. But I have followed Fleming's correction, to respect the concept of conditional probability justifying eq. 3.3.
} 
that the conditional probability eq. 3.6 is equal to

$$
\mathcal{P}\left(O_{0} \mid T\right):=\frac{\operatorname{Tr}\left(P_{O_{0}}(0) \rho_{\text {eff }}(T)\right)}{\operatorname{Tr}\left(\rho_{\text {eff }}(T)\right)}:
$$

which is of the familiar form, as in eq. 3.4, except with the effective density matrix $\rho_{\text {eff }}(T)$ substituted for the background-time density matrix $\rho(t)$. Thus the definition of $\rho_{\text {eff }}(T)$ makes eq. 3.6 take the familiar form eq. 3.4.

To sum up: the statistics we gather for the quantity $\hat{O}$ at the clock time $T$ for a system prepared at $t=0$ in $\rho_{\text {sys }}$ are given by a familiar-looking formula, viz. eq. 3.10: but one which uses the density matrix $\rho_{\text {eff }}(T)$ defined in eq. 3.8. Looking at that definition, we infer: the statistics are as if the system is drawn from an ensemble, whose components are (i) indexed by $t \in \mathbb{R}$, (ii) given by $U_{\text {sys }}(t) \rho_{\text {sys }} U_{\text {sys }}(t)^{\dagger}$ and (iii) weighted with the probability density $\mathcal{P}_{t}(T)$ as given by eq. 3.7.

The physical idea is clear, and natural. Because the clock may be inaccurate, i.e. $T$ may be unequal to $t$, the statistics at a given $T$ are a mixture of the orthodox statistics associated with unitary evolutions to various times $t$, with the mixture's weights being given by $\mathcal{P}_{t}(T)$, the probability density at given $T$ of the component labelled $t$. Thus the lab technician might report: 'I measure $O$ when the clock reads ' $T$ '. But the statistics of $O$ that I gather are as if each measurement draws the system from an urn, in the state $U_{\text {sys }}(t) \rho_{\text {sys }} U_{\text {sys }}(t)^{\dagger}$ with a probability $\mathcal{P}_{t}(T) d t^{\prime}$.

It is also clear that in terms of the clock-time $T$, evolution is not unitary: for $\rho_{\text {eff }}(T)$ is a convex combination of density matrices, each of which is unitarily evolving with respect to $t$, but which are associated with different values of $t$. This temporal decoherence will be at the centre of the Montevideo interpretation.

The Montevideans write down the equation of motion for $\rho_{\text {eff }}(T)$ in terms of $T$, on the assumption that the clock is "good", in the sense that the probability density $\mathcal{P}_{t}(T)$ takes the form

$$
\mathcal{P}_{t}(T)=f(T-t)
$$

where $f$ achieves a maximum at $T=t$, and has narrow spread (i.e. decays rapidly, away from $T=t$ ). As one would guess, the equation (derived by considering a Taylor expansion around $t$ ) is like the usual quantum Liouville equation for a Schroedingerpicture density matrix, but with additional terms. I will not need its exact form; (technically, it is a Lindblad equation, cf. e.g. eq. 14 of Gambini, Porto and Pullin (2007)). But I do need a solution of it. Namely: if one assumes that the the distribution $f$ is symmetric and its width grows linearly with respect to clock-time $T$, then the equation can be exactly solved; and if the system has a discrete energy spectrum, the solution is

$$
\rho_{\text {eff }}(T)_{n m}=\left[\rho_{\text {eff }}(0)_{n m}\right] \exp \left(-i \omega_{n m} T\right) \exp \left(-\sigma \omega_{n m}^{2} T\right)
$$

where: (i) $\omega_{n m}:=\omega_{n}-\omega_{m} \equiv\left(E_{n}-E_{m}\right) / \hbar$ is the difference of the frequencies for levels $n$ and $m$; and (ii) $\sigma$ is the (constant) rate of change, with respect to $T$, of the width of $f$. Thus the off-diagonal terms go to zero exponentially. 
The first comment to make about the analysis just summarized is that it is clear and uncontentious. For it is an orthodox quantum-theoretic analysis, with a natural physical idea underlying its effective density matrix $\rho_{\text {eff }}(T)$. Besides, the Montevideans note that some other analyses, with different motivations than theirs, get the same or a similar result; and that the result successfully describes some real experiments with appropriately 'bad' clocks $\hat{T}$. But as one would expect, it is unclear and even contentious how the mixture eq. 3.8, or its special case eq. 3.12, can contribute to solving the measurement problem - cf. the next Section.

\section{The meanings and roles of a mixture}

In this Section, I will argue that the mixture in the general form, eq. 3.8, does not fulfill the usual two roles for which a mixture is obtained, or hoped for, in approaches of the quantum measurement problem; while on the other hand, the special case eq. 3.12 does fulfill the first role. I will do this by first defining the roles, together with two familiar approaches, viz. decoherence by the environment and dynamical reduction (Section 4.1). The situation for the Montevideans' mixtures will then be clear, in Section 4.2.

I stress that this contrast, as to fulfillment of the two roles, will not be an objection to Section 3's analysis; nor even to the Montevideans' proposed use of it to solve the measurement problem. The basic reason why not is simply that the Montevideans do not ask temporal decoherence to fulfill the usual two roles for a mixture. Rather, they propose that it, especially the mixture's special form eq. 3.12, answers objections made to environment-induced decoherence - which they accept as playing a crucial part in solving the measurement problem. This proposal, appealing to considerations of quantum gravity, will be the topic of Section 5. But we will get a clearer view of the proposal, by first spelling out this contrast about the two roles.

\subsection{Two roles, two approaches and two debates}

Thus consider the two roles that a mixture, obtained in an analysis of quantum measurement, is usually taken as needing to fulfill. I give them obvious labels:

(Basis): To define a preferred quantity, or basis, or set of nearly orthogonal states: and usually, one wants this quantity to be one that intuitively is definite at the end of measurement (e.g. a quantity such as the position of the centre of mass of a pointer) . This role - or the goal, or difficulty, of fulfilling it - is sometimes called 'the preferred basis problem', or 'the basis problem'.

(Outcome): To secure that in each specific measurement trial, there is an individual outcome (represented mathematically by one component of the mixture). This roleor the goal, or difficulty, of fulfilling it - is sometimes called 'the problem of outcomes'; or following d'Espagnat's jargon (1976, Section 6.6), 'the problem of interpreting the mixture as proper (i.e. ignorance-interpretable) rather than improper (obtained by 
partial tracing)'; or following Bell's jargon, 'the problem of replacing 'and' by 'or'. (The Montevideans use all three labels.)

Broadly speaking, (Outcome) presupposes (Basis): only once a quantity or basis is preferred, can one raise the question of securing one of the quantity's values, or one of the basis' elements.

To illustrate what is at stake in fulfilling these roles, let us consider two familiar approaches to the measurement problem - which, as mentioned, will help us to 'locate' the Montevideo interpretation. Again, I use labels:

(Ein): Decoherence by the environment, as studied by the Heidelberg school of Zeh and co-authors, and the Los Alamos school of Zurek and co-authors; (cf. Giulini et al. (1996), Schlosshauer (2008). My label 'Ein' abbreviates 'einselection', a word invented by Zurek to convey 'environment-induced superselection'.

(DRP): The dynamical reduction programme of Ghirardi, Rimini, Weber, Pearle and others (begun, in large measure, by Ghirardi et al. (1986)). This programme models the collapse of the wave packet (Section 2.1.1) as a physical process.

Without going into details, I first report what I take to be the literature's consensus, which the Montevideans (and I) endorse. Namely:

(Ein) fulfills (Basis): for decoherence selects a quantity like the position of the centre of mass of the apparatus pointer, in the sense that after the decoherence process, the state of the apparatus and measured system is a density matrix that is nearly diagonal in that quantity.

But (Ein) does not fulfill (Outcome). This is expressed in various ways by various authors; cf. e.g. Giulini et al (1996, p. 37) and Leggett (2008). But the main point is that since the density matrix has been obtained by tracing over the environment, it represents an improper, not proper, mixture: there is no selection (even in some conceptual sense) of one of its components as against the others. Indeed, the interference terms, that are characteristic of the total system (including the environment) being in a pure state (and thus symptomatic of the mixture being improper), can in principle be revealed by measuring a suitable quantity on the total system (a 'global quantity').

(DRP), on the other hand, fulfills both (Basis) and (Outcome). It fulfills (Basis) by construction. For the revision of the Schroedinger equation explicitly exhibits the quantity (typically defined in terms of position and mass-density) with respect to which the final density matrix of the apparatus and measured system is nearly diagonal. And (Outcome) is fulfilled because this density matrix represents a proper, i.e. ignorance-interpretable, mixture: for it has not been obtained by tracing over the environment. Besides, (DRP)'s scheme contains two other mathematical representations of the individual outcome of a specific measurement trial: viz. a much higher amplitude for that outcome than for the others, and an associated realization (also known as: trajectory) of the postulated stochastic process which determines the outcome in question; (whereas (Ein) has no such higher amplitude for a single outcome, and no such stochastic process).

Agreed, that is a hasty summary. There are various debates about whether (Ein) 
and (DRP) can succeed. To prepare for the Montevideans' proposals in Section 5, I need to note two such debates.

\subsubsection{Can we ignore small amplitudes?}

I just said that (Ein) and (DRP) each fulfill (Basis) by securing a final density matrix of the apparatus and measured system which is nearly diagonal in a quantity that intuitively one wants to be definite. But one common line of objection to (Ein) and (DRP) presses the question: is 'nearly' good enough? The off-diagonal terms of the density matrix may have a tiny modulus, but they are physically real.

Here we should distinguish three issues (and maybe more!). The first two apply, I believe, to both (Ein) and (DRP); the third, usually called the 'problem of tails', applies only to (DRP). I only need to state them: I will not need to pursue them (thank goodness!).

(i): Revivals: In some models, after a very long time the off-diagonal terms become large: so that whatever definiteness of the preferred quantity we had won by the end of the measurement interaction turns out in the very long run to have been but a temporary victory. This is called the problem of revivals (or 'recurrence of coherence', or 'recoherence').

(ii): Which quantity?: One natural tactic for replying is to say that we should not be dogmatic ab initio about exactly which quantity is to be secured as definite, in order to solve the measurement problem. We should be flexible: if calculations suggest that a quantity different from the one we at first wanted definite, but in some sense close to it, is definite - then we should be satisfied with that. Thus suppose the density matrix at the end of the measurement interaction is nearly diagonal in the quantity, $\hat{Q}$ say, that we at first wanted definite, but is exactly diagonal in a quantity $\hat{Q}^{\prime}$, with $\hat{Q}^{\prime}$ and $\hat{Q}$ suitably close (say, in operator norm). Then that is surely good enough: at least for early times - since we must admit this tactic does not answer the problem of revivals.

(iii): Tails: Finally, small-amplitude terms have been taken as an objection to (DRP)'s claim to have fulfilled (Outcome) by the final state having a much higher amplitude for some single outcome than for the others. Thus some people object that a possible outcome having a large, rather than tiny, amplitude is no rationale for deeming it, rather than the other outcomes, to have occurred. Why not say instead that any outcome with a non-zero, albeit tiny, amplitude occurs? (This is called the problem of tails.)

\subsubsection{Is vagueness a virtue or a vice?}

Section 4.1.1's debate about small amplitudes is part of a larger debate about whether a solution to the measurement problem must make an absolutely precise statement about which quantities on which systems have definite values (and with which probabilities).

On the one hand, the tactic (ii) in Section 4.1.1 suggests we can be, even should 
be, flexible. Thus we require a solution to the measurement problem to vindicate the classical physical description ('the appearance of a classical world', as it is sometimes put) - but only approximately. Only some subset of quantities should have definite values. And maybe that subset should only be specified contextually, even vaguely. And maybe the values should only be definite within some margins of error, even vague ones. Clearly, (Ein) secures this sort of flexibility. For the selection of the preferred quantity is made by a dynamical process - whose definition can be legitimately varied in several ways. Three examples: the definitions of:

(a) the system-environment boundary (and therefore of what exactly is the interaction hamiltonian), and of

(b) the time at which the interaction ends, and of

(c) what counts as a state being 'sharply peaked' for a quantity, can all be varied. Of course, item (c) echoes Section 4.1.1's debate about small amplitudes. And (DRP) shares with (Ein) the flexibility about defining (b) and (c); but not (a), since it makes no system-environment split.

On the other hand, there is a strong impulse to require precision; for (at least) two reasons. First: over the eight decades since the measurement problem was first articulated, many proposals for how to solve it have been vague in ways that undermined the proposal: the vagueness amounted to hand-waving (as the writings of Bell often lamented). Second: most philosophers construe vagueness as semantic indecision. That is: our words and concepts being vague is a matter of our not having decided on a precise boundary between instances and non-instances - and not a matter of reality itself being vague (a notion which may well not be coherent). Most philosophers also take mathematics to be precise, i.e. not vague. They also take physics to be in large part precise, often because it is expressed in mathematical language. And where it is not precise (as in much heuristic physics), they take it nevertheless to aspire to precision. So they would naturally require quantum theory, in purporting to be a fundamental theory, to give, or aspire to give, a precise i.e. non-vague solution to its measurement problem.

I will return to this debate, and my own take on it, in Section 5.

So much by way of summarizing, partly in preparation for Section 5: the two usual roles for a mixture, how the two approaches (Ein) and (DRP) treat those roles, and the debates about their treatments. In the light of those debates, I should perhaps weaken what I called 'the literature's consensus' about these approaches' fulfilling the roles, and speak only of aims. That is: a more cautious summary would be to say just that (Ein) aims to fulfill (Basis), but does not aim to fulfill (Outcome); while (DRP) aims to fulfill both (Basis) and (Outcome).

In any case, this summary is enough for my purposes. For we can now check whether the Montevideo interpretation's temporal decoherence mixtures, eq. 3.8 and eq. 3.12, fulfill the roles (Basis) and (Outcome). 


\subsection{Montevideo's mixtures}

I begin with the first, general form, eq. 3.8. Clearly, it does not fulfill the roles (Basis) and (Outcome). As to (Basis), eq. 3.8 does not suggest an orthogonal, or nearly orthogonal, decomposition of $\rho(T)$ even if the initial state $\rho_{\text {sys }}$ is pure (given as $|\psi\rangle\langle\psi|$ ). For of course typical components labelled by different values of $t, U_{\text {sys }}\left(t_{1}\right) \rho_{\text {sys }} U_{\text {sys }}\left(t_{1}\right)^{\dagger}$ and $U_{\text {sys }}\left(t_{2}\right) \rho_{\text {sys }} U_{\text {sys }}\left(t_{2}\right)^{\dagger}$, will not be orthogonal. Looking ahead for a moment: this aspect will remain when, in Section 5, we 'throw away the ladder' of assuming a background time $t$.

As to (Outcome), the situation is a little subtle. I said that (Outcome) presupposes (Basis): so since (Basis) is not fulfilled, (Outcome) can hardly be. But on the other hand, I stress that since we are at present assuming that there is a background time $t$, each individual system measured at clock time $T$ has in fact a time $t$ : i.e. has in fact persisted from the initial time $t=0$ to a time $t$, at which it is measured for $O$ and the clock says ' $T$ ' (with maybe $T \neq t$ !). In short: though the value of $t$ does not deserve the name 'outcome' (which presumably should here be reserved for entities correlated with values of the quantities $\hat{O}$ and $\hat{T}$ ), this value is perfectly definite in each specific measurement trial. But as one would guess: this 'definiteness of $t$ ' will disappear when, in Section 5, we 'throw away the ladder' of assuming a background time $t$.

I turn to the special case, eq. 3.12. This is nearly diagonal in a basis that is of central physical significance, the energy basis. So the situation is obviously similar to that which occurs in decoherence by the environment. That is: eq. 3.12 fulfills the first role, (Basis). It selects energy as the preferred quantity: of course, modulo the debate in Section 4.1.1, about whether we may ignore small amplitudes - in particular the problem of revivals. And eq. 3.12 does not fulfill, and does not aim to fulfill, the second role, (Outcome): no component of the mixture is selected.

In the light of this discussion, it is natural to ask how if at all the Montevideo interpretation will fulfill (Basis) and (Outcome). As I see matters, the short answerto be expanded in Sections 5 and 6 - is as follows.

As to (Basis): The interpretation appeals to a combination of (Ein) and temporal decoherence, in the specific form of eq. 3.12. More specifically, temporal decoherence is proposed as solving the problem of revivals that besets (Ein)'s fulfillment of (Basis); (cf. Section 4.1.1). The idea will be that temporal decoherence makes revivals unobservable in principle, not merely in practice.

As to (Outcome): The situation is less clear to me. To explain this, I should distinguish two aspects of (Outcome) which I so far have not needed to separate:

(i) justifying the ascription of a proper (i.e. ignorance-interpretable) mixture;

(ii) justifying the ascription to an individual system of a single component of such a mixture.

As I see matters, the Montevideans succeed in (i). They give details about how temporal decoherence, especially eq. 3.12, answers the usual 'global quantity' objection that, as mentioned in Section 4.1, prevents (Ein) fulfilling (Outcome). Their idea will 
be similar to their idea just above, for (Basis): temporal decoherence makes the global quantity unmeasurable in principle, not merely in practice.

But what about (ii): securing an individual outcome in each specific measurement trial? Here, I think the situation is less clear. I myself think their interpretation fits best in an Everettian picture, for reasons to do with the vagueness debate of Section 4.1.2. But some of their remarks suggest they disagree. But as I mentioned, Sections 5 and 6 will expand on this.

\section{Throwing away the ladder}

The title refers to Wittgenstein's metaphor of throwing away a ladder once one has climbed it to gain an understanding. Here, the ladder is the background time $t$ : and nowadays the various research programmes seeking a quantum theory of gravity make rival proposals for how to throw away this ladder. As mentioned in Section 2.1.3, the Montevideans work in one such programme, viz. quantum general relativity; but the Montevideo interpretation does not depend on its details. We can state their interpretation as five proposals. The first four proposals are treated in Section 5.1. The fifth proposal is separated out, in Section 5.2, since it leads in to Section 6's concluding suggestion that the Montevideo interpretation is best seen as Everettian.

\subsection{Four proposals}

The Montevideans propose that:

(i): Some heuristic arguments about black holes suggest that there are fundamental limits to how accurate a quantum clock can be, so that the temporal decoherence of Section 3 cannot be completely eliminated.

(ii): The equation of motion in terms of a clock-quantity $T$ for a clock that is as accurate as possible should be taken as fundamental. This is the 'throwing away of the ladder' of background time $t$. So the ineliminable decoherence is now a fundamental or principled type of decoherence.

(iii): This fundamental decoherence, together with environmental decoherence, solves the quantum measurement problem, along the lines given in Section 4.2.

(iv): Besides, although the arguments in (i) vary somewhat in the accuracy limits they suggest, the ensuing solution to the measurement problem is robust: it holds good if one adopts the other accuracy limits.

I will present (i)-(iv), in order (Sections 5.1.1 to 5.1.4). But my discussion will be much briefer than in Sections 3 and 4. This reflects not just lack of space: also, the authors' arguments are more heuristic; and so my own grip on them is more tentative. These technicalities will lead in to the Montevideans' final interpretative proposal, about under what exact conditions 'the wave-packet collapses' (Section 5.2)-which will prompt me to suggest in Section 6 that the interpretation fits best in an overall 
Everettian approach.

\subsubsection{Heuristic arguments}

As I announced in (ii) of Section 2.2, the Montevideans endorse arguments by various authors, especially Salecker and Wigner, and Ng and van Dam, suggesting fundamental limits on quantum clocks. Thus Salecker and Wigner argued that the mass of a clock limited its accuracy: specifically, if $T$ is the time-interval to be measured by a clock of mass $M$, then there must be an uncertainty $\delta T$ proportional to $\sqrt{ } T / M(1957$, pp. 572 574; cf. also Wigner (1957, pp. 260-61)). This suggests that one can attain arbitrary accuracy by a sufficiently massive clock.

But Ng and van Dam (1994) argued that in general the mass needs to be confined; and that therefore the attainable mass, and so accuracy, is limited by the onset of the formation of a black hole. According to their analysis:

(a) if $T$ is again the time-interval to be measured, the maximum attainable mass is given by the mass of a black hole whose Schwarzschild radius is the distance light would travel in $T$; and

(b) the corresponding maximum attainable accuracy is given by an uncertainty $\delta T$ proportional to $T_{\text {Planck }}^{2 / 3} T^{1 / 3}$, where $T_{\text {Planck }}=10^{-44}$ seconds is the Planck time. So for typical laboratory times of a few hours, $\delta T \sim 10^{16} T_{\text {Planck }} \sim 10^{-28}$ seconds. This is not now experimentally measurable, but in principle it could be observed.

This analysis remains heuristic, indeed apparently controversial; (cf. the exchange between Baez and Olson (2002) and Ng and van Dam (2003)). But the Montevideans endorse this kind of analysis: taking heart from the fact (Section 5.1.4) that their solution to the measurement problem seems insensitive to what are the exact limits on clocks' accuracy.

\subsubsection{Fundamental decoherence}

Prompted by the arguments in Section 5.1.1, the Montevideans propose to dispense with the time $t$ that formed the background of Section 3's analysis: the fundamental equation of motion is to be their equation of motion in terms of $T$, the clock-quantity for a maximally accurate clock.

Or rather, to be precise: they take the arguments in Section 5.1.1 to suggest that the unknown theory of quantum gravity, whatever its exact form, will yield their equation of motion using this $T$, as the effective equation of motion governing a regime, whose exact definition we of course cannot give (being ignorant of the quantum gravity theory!) but which we expect to include all the successful applications of quantum theory, both non-relativistic and relativistic, "away from" quantum gravity effects such as might occur in black holes. ${ }^{9}$

\footnotetext{
${ }^{9}$ Like almost all the literature on decoherence, the Montevideans' analyses look non-relativistic;
} 
This proposal implies that attention focusses on Section 3's exact solution to their equation, for a system with discrete energy levels, eq. 3.12, but now with the width $\sigma(T)$ given as $\left(\frac{T_{\text {Planck }}}{T}\right)^{1 / 3} \cdot T_{\text {Planck }}$, i.e.

$$
\rho_{\text {eff }}(T)_{n m}=\left[\rho_{\text {eff }}(0)_{n m}\right] \exp \left(-\omega_{n m} T\right) \exp \left(-\omega_{n m}^{2} T_{\text {Planck }}^{4 / 3} T^{2 / 3}\right) .
$$

\subsubsection{Fundamental decoherence allied with environmental decoherence}

We can now sketch how eq. 5.1 is meant to supplement the process of environmental decoherence (called (Ein) in Section 4.1), so as to solve the measurement problem. As I announced in Section 4.2, the idea is that this fundamental temporal decoherence kills the off-diagonal terms of the density matrix of the macroscopic apparatus (together with the measured system). In particular, this solves:

(i): the problem of revivals, i.e. the fact that in some models without temporal decoherence, the off-diagonal terms can in principle, in the very long run, become large. (In terms of Section 4.1.1's debate about small amplitudes: this problem confronts (Ein)'s fulfillment of the role (Basis).)

(ii): the problem of global quantities, i.e. the fact that in some models without temporal decoherence, one can in principle measure a quantity whose statistics reveal the total system, including the environment, to be in a pure (entangled) state. (In terms of Section 4.1: this problem confronts (Ein)'s fulfillment of the role (Outcome).)

Here is a bit more detail about (i); (I set aside (ii), for which the discusssion is similar). The general idea is that without temporal decoherence, an off-diagonal term is a product of $N$ complex numbers of unit modulus $\exp \left(i\left(\theta_{k} t+a_{k}\right)\right), k=1, \ldots, N$, where $N$ represents the number of degrees of freedom of the apparatus (and measured system), and so is typically very large, and the phases $a_{k}$ are randomly distributed. Because $N$ is large, the common period of oscillation for the terms is very long, maybe even comparable with the age of the universe - yet nevertheless finite. But when we include temporal decoherence in the calculation, there is an extra exponential suppression. Thus one detailed analysis (Gambini, Garcia Pintos and Pullin, 2010, Sections IV-V) studies a spin model of Zurek's, and a more realistic modification of it. Owing to the temporal decoherence, the off-diagonal terms get multiplied by an $N$-fold product of exponentials whose exponents are proportional to $T^{2 / 3}$. Putting in the figures for the models, this gives unobservably small tails, and so no revivals even in very long times, even for $N$ between 100 and 1000 .

\subsubsection{Robustness}

In Section 5.1.1, I reported that heuristic arguments suggest that the most accurate clock has an uncertainty $\delta T$ proportional to $T_{\text {Planck }}^{2 / 3} T^{1 / 3}$. Here we should note that the

but one can imagine generalizing them to get an explicit Lorentz-invariance. Recall Section 2.1.3's contrast between general relativity's treating time as dynamical, and quantum theory's treating it as 'background', as in the cousin classical, Newtonian or special-relativistic, theories. 
Montevideans admit that some arguments suggest instead that $\delta T \sim T_{\text {Planck }}^{1 / 2} T^{1 / 2}$.

But this makes essentially no difference to their conclusions, or even to their quantitative results in Section 5.1.3, about their proposed solution to environmental decoherence's problems of revivals and global quantities. For provided that the uncertainty in time goes as a positive power of $T$, one loses coherence. And quantitatively, as regards the solution to the problem of revivals, reported in Section 5.1.3: if one were to postulate more cautiously that the uncertainty goes like a small positive power, i.e. $\delta T \sim T_{\text {Planck }}^{1-\varepsilon} T^{\varepsilon}$ for some small $\varepsilon$, then the only effect would be that the value of $N$ required to prevent revivals even in very long times would be raised from $N \sim 100$ to about $N /(3 \varepsilon)$.

\subsection{The fifth proposal: about exactly when is there an out- come}

So much by way of reporting the four proposals. At this point, one naturally wants to press the following straightforward point:

Temporal decoherence involves exponentials: its suppression of interference is by dictating that exponential factors should multiply the terms that in environmental decoherence remain troublesome. So the trouble persists. That is: the terms remain, generically, non-zero for arbitrarily large times etc. So, presumably, the wave-function does not in fact collapse. More precisely: these four proposals have not secured that in each specific measurement trial, there is an individual outcome. In short: we have not yet passed from Bell's 'and' to his 'or'.' To put the point in Section 4.1's jargon about the roles that a mixture might fulfill: the four proposals have not fulfilled the role (Outcome).)

The Montevideans are of course aware of this point. In answer, they make the following interpretative proposal, which completes their exposition. (At least: it completes it, in the order of exposition I have adopted in this paper.)

They say: there is transition from 'and' to 'or' — an individual outcome, a selection of a single component of the mixture, an event - exactly when it becomes in principle undecidable (thanks to fundamental temporal decoherence) whether or not the evolution of the total system is unitary. Here, philosophers will find the word 'undecidable' reminiscent of logic and computability theory; but these connotations are not intended. All that is intended is just that no measurement of any quantity, even in principle, can ascertain whether the evolution is unitary. Thus for a statement of this proposal, cf. Gambini, Garcia Pintos and Pullin (2010, Section III and Appendix 2.A), Gambini and Pullin (2009: replies to questions 10 and 11). They develop the proposal in more detail, for their modification of Zurek's spin model (mentioned at the end of Section 5.1.3), in Gambini, Garcia Pintos and Pullin (2011, Sections 3-5). I will not enter into details, but just make two points: the second leading in to Section 6 . 
First: this last discussion builds on some remarkable in-principle limitations on measuring spins proposed by Kofler and Brukner (2010). Second: nevertheless, the undecidability proposal about exactly when is the collapse, remains, in the Montevideans' publications so far, tied to specific models - prompting the question 'What is their exact and general proposal?'

\section{Everett en fin}

Here, I suggest, we see at last that the Montevideo interpretation fits best in an Everettian approach, in which a 'branching' - in the above jargons: a transition from 'and' to 'or', an outcome, a selection, an event - is effective and approximate. And this returns us (as I promised) to the debate in Section 4.1.2, about whether vagueness is a virtue or a vice.

For of course, in the usual Hilbert space formalism, any pure state and any mixed state are distinguished by the statistics, according to the Born rule, of some self-adjoint operator or other. So a rigorous statement of undecidability in the Montevideans' sense would involve, just as a matter of logic, a rigorous statement of what is the proposed restriction on quantities: which the Montevideans have not so far given. ${ }^{10}$

Thus as I see matters, the Montevideans face a strategic choice in developing their interpretation:

(1): If they feel obliged to make such a statement: then their interpretation, though undoubtedly already of great interest, is not yet complete. Of course, incompleteness is no discredit: especially since one might think we should wait for the heuristic gravitational arguments in Section 5.1.1 to be made rigorous and-or to be extended, so as to fill out the arguments of Sections 5.1.2 and 5.2.

(2): If they do not feel so obliged: then this should be - I suggest-because they view such vagueness as a virtue, not a vice.

And as discussed in Section 4.1.2, such vagueness is indeed a virtue on an Everettian approach, according to which the 'branching' is phenomenological or effective in physicists' sense. That is: branching is an approximation whose value does not depend on its being precisely defined - indeed, the value depends on its not being precisely defined. We saw there how environmental decoherence secured the desired flexibility about which quantities have definite values, by its flexibility in the definitions of:

(a) the system-environment boundary,

(b) the time at which the decohering interaction ends, and

(c) what counts as a state being 'sharply peaked'.

We can now add that the Montevideans' temporal decoherence has similar flexibility about (b) and (c). Thus I suggest that if the Montevideans choose (2), then their

\footnotetext{
${ }^{10}$ Though this sort of rigorous statement is often made by those adopting the algebraic approach to quantum theory, such statements can of course be made using the Hilbert space formalism: which is the formalism the Montevideans adopt.
} 
interpretation fits best with an Everettian picture of 'effective branching'.

Think of how valuable we find it to describe the region where a river meets the sea in terms of a delta composed of islands and channels. We do not need to decide what precisely counts as a channel, or a fork in the waterway. Indeed the description is more valuable precisely because we do not decide: to give precise definitions would be a waste of time, and would make our language and thought prolix and inflexible, and so less adaptable to the demands of a passing occasion. The helmsman who asks 'Shall I take the left or the right channel?' would be annoyed to be told 'It depends on how one defines 'main channel".

But in closing, I stress that my overall aim here has been, not to object to the interpretation - but to praise it! It has the merit of bringing specific, albeit heuristic, considerations of quantum gravity to bear on the measurement problem. So I invite the reader to explore it.

Acknowledgements:- For comments on a previous (2012) version, I am very grateful to: Gordon Fleming, Rodolfo Gambini, Jorge Pullin, David Sloan, James Yearsley, David Wallace and Chris Wüthrich; and to audiences in Dubrovnik, Croatia, and Cambridge and Oxford, UK. My thanks also to the editor and two referees of their comments. This work is also supported in part by a grant from the Templeton World Charity Foundation, whose support is gratefully acknowledged.

\section{References}

Baez, J. and Olson, S (2002), 'Uncertainty in measurements of distance', Classical and Quantum Gravity 19 L121; arxiv: gr-qc/0201030v1

Bell, J. (1986), 'Six possible worlds of quantum mechanics', Proceedings of the Nobel Symposium 65 (Stockholm August 1986); reprinted in Bell (1987), page references to reprint.

Bell, J. (1987), Speakable and Unspeakable in Quantum Mechanics, Cambridge: Cambridge University Press; second edition 2004, with an introduction by Alain Aspect.

Busch, P. (1990), 'On the energy-time uncertainty relation: Part I: Dynamical time and time indeterminacy', Foundations of Physics 20, pp. 1-32.

Busch, P. (1990a), 'On the energy-time uncertainty relation: Part II: Pragmatic time versus energy indeterminacy', Foundations of Physics 20, pp. 33-43.

Busch, P. (2008), 'The time-energy uncertainty relation', Chapter 3 of Time in Quantum Mechanics, ed. G. Muga et al., Springer Verlag (second edition, 2008); quant-ph/0105049. 
Butterfield, J. (2002), 'Some Worlds of Quantum Theory', in R.Russell, J. Polkinghorne et al (ed.), Quantum Mechanics (Scientific Perspectives on Divine Action vol 5), Vatican Observatory Publications, 2002; pp. 111-140. Available online at: arXiv.org/quant-ph/0105052; and at http://philsci-archive.pitt.edu/archive/00000204.

Butterfield, J. (2012), 'On time in quantum physics', in The Blackwell Companion to Time, edited by A. Bardon and H. Dyke; Oxford: Blackwell; pp. 220-241; available at http://philsci-archive.pitt.edu/9287/

Butterfield, J. and Isham C. (2001), 'The philosophical challenge of quantum gravity', in C. Callender and N. Huggett (eds.), Physics Meets Philosophy at the Planck Scale, Cambridge University Press, pp. 33-89; gr-qc/9903072;

http://philsci-archive.pitt.edu/1915/

Dennett, D. (1991), 'Real patterns', Journal of Philosophy, 88, pp. 27-51.

d'Espagnat, B. (1976), Conceptual Foundations of Quantum Mechanics, Reading, MA: W. A. Benjamin

Deutsch, D. (2010), 'Apart from universes', in Saunders et al (eds.) (2010), pp. $542-552$.

Gambini, R., Garcia Pintos, L. and Pullin J. (2010), 'Undecidability and the problem of outcomes in quantum measurements', Foundations of Physics 40, pp. 93-115; arXiv:0905.4222

Gambini, R., Garcia Pintos, L. and Pullin J. (2011), 'Undecidability as solution to the problem of measurement: fundamental citerion for the production of events', International Journal of Modern Physics D20, pp. 909-918; arXiv:1009.3817

Gambini, R., Garcia Pintos, L. and Pullin J. (2011a), 'An axiomatic formulation of the Montevideo interpretation of quantum mechanics', Studies in the History and Philosophy of Modern Physics 42, pp. 256-263; arXiv:1002.4209v2

Gambini, R., Porto R. and Pullin J. (2006), 'Fundamental spatiotemporal decoherence: a key to solving the conceptual problems of black holes, cosmology and quantum mechanics', International Journal of Modern Physics D15, pp. 2181-2186; arXiv:grqc/0611148

Gambini, R., Porto R. and Pullin J. (2007), Fundamental decoherence from quantum gravity: a pedagogical review', General Relativity and Gravitation 39, pp. 11431156; arXiv:gr-qc/0603090

Gambini, R., Porto R. and Pullin J. (2008), 'Loss of entanglement in quantum mechanics due to the use of realistic measuring rods', Physics Letters A 372, pp. 1213-1218; arXiv:0708.2935

Gambini, R., Porto R., Pullin J. and Torterolo S. (2009), 'Conditional probabilities with Dirac observables and the problem of time in quantum gravity', Physical Review D79, pp. 041501; arXiv:0809.4235v1

Gambini, R. and Pullin J. (2007), 'Relational physics with real rods and clocks 
and the measurement problem of quantum mechanics',Foundations of Physics 37, pp. 1074-1092; arXiv: quant-ph/0608243

Gambini, R. and Pullin J. (2009), 'The Montevideo interpretation of quantum mechanics: frequently asked questions' Journal of Physics Conference Series, proceedings of the DICE 2008 Castiglioncello meeting, 174, 012003; arXiv:0905.4402.

Gambini, R. and Pullin J. (2009a), 'Free will, undecidability and the problem of time in quantum gravity' Essay for FQXi; arXiv:0903.1859 [quant-ph].

Ghirardi, G., Rimini, A. and Weber, T. (1986), 'Unified dynamics of microscopic and macroscopic systems', Physical Review D34, pp. 470-491.

Giulini, D., Joos, E., Kiefer, C., Kupsch J., Stamatecu I. and Zeh. H (1986), Decoherence and the Appearance of a Classical World in Quantum Theory, Berlin: Springer.

Kent, A. (2010), 'One world or many?' in Saunders, Barrett, Kent and Wallace (Eds.) (2010), pp. 307-354.

Kofler, J. and Brukner C. (2010), 'Are there fundamental limits for observing quantum phenomena from within quantum theory?'; arXiv: 1009.2654

Leggett, A. (2008), 'Realism and the physical world', Reports on Progress in Physics, 71, 022001; doi:10.1088/0034-4885/71/2/022001

Messiah, A. (1965), Quantum Mechanics, volume 1, North Holland.

Muga, J., Sala Mayato, R. and Egusquiza I. (eds.) (2008), Time in Quantum Mechanics, Berlin; Springer, two volumes.

Ng, Y. and van Dam, H. (1994), Modern Physics Letters A9, pp. 335-340.

Ng, Y. and van Dam, H. (2003), 'Comment on 'Uncertainty in measurements of distance", Classical and Quantum Gravity, 20, p. 393; arxiv: gr-qc/0209021.

Rovelli, C. (2006), 'Quantum gravity', in J. Butterfield and J. Earman (eds.), Philosophy of Physics, in the series The Handbook of Philosophy of Science, Amsterdam: Elsevier, pp. 1287-1330.

Salecker, H. and Wigner, E. (1957), 'Quantum limitations of the measurement of spacetime distances', Physical Review 109, pp. 571-577.

Saunders S., Barrett J., Kent A. and Wallace D. (eds.) (2010), Many Worlds? Everett, Quantum Theory and Reality, Oxford: Oxford University Press.

Schlosshauer, M. (2008), Decoherence and the quantum to classical transition, Berlin: Springer.

Wallace, D. (2001), 'Worlds in the Everett interpretation', Studies in the History and Philosophy of Modern Physics 33, pp. 637-661.

Wallace D. (2012), The Emergent Multiverse, Oxford: Oxford University Press.

Wallace, D. (2012a), 'Decoherence and its role in the modern measurement prob- 
lem', Philosophical Transactions of the Royal Society of London A 370, pp. 4576-4593; http://uk.arxiv.org/abs/1111.2187

Wigner, E. (1957), 'Relativistic invariance and quantum phenomena', Reviews of Modern Physics 29, pp. 251-268.

Yearsley, J. (2011), 'Aspects of time in quantum theory', arxiv: 1110.5790 